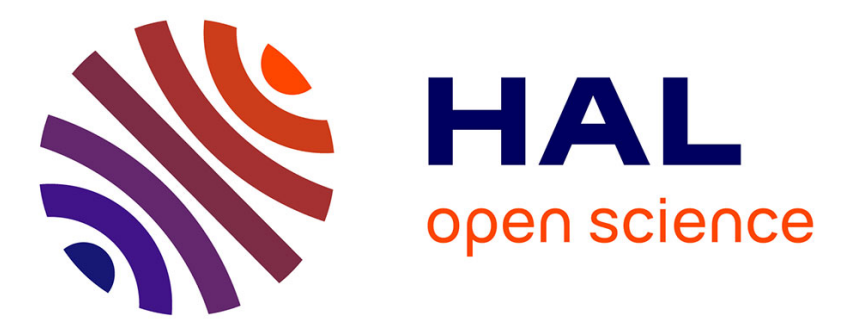

\title{
Répondre à la (question de la) structure, en contexte de maladie génétique \\ Olivier Putois
}

\section{To cite this version:}

Olivier Putois. Répondre à la (question de la) structure, en contexte de maladie génétique. Figures de la psychanalyse, 2019, nº 38 (2), pp.191-198. 10.3917/fp.038.0191 . hal-03005206

\section{HAL Id: hal-03005206 https://hal.science/hal-03005206}

Submitted on 13 Nov 2020

HAL is a multi-disciplinary open access archive for the deposit and dissemination of scientific research documents, whether they are published or not. The documents may come from teaching and research institutions in France or abroad, or from public or private research centers.
L'archive ouverte pluridisciplinaire $\mathbf{H A L}$, est destinée au dépôt et à la diffusion de documents scientifiques de niveau recherche, publiés ou non, émanant des établissements d'enseignement et de recherche français ou étrangers, des laboratoires publics ou privés. 


\title{
Répondre à la (question de la) structure, en contexte de maladie génétique
}

\author{
Olivier Putois
}

D'un point de vue épistémologique, l'entente freudienne de la notion de structure se saisit dans le dépassement de ce que l'épistémologie générale appelle "réalisme naïf »: si elle dessine les arêtes du réel clinique propre à chaque cas, la structure n'apparaît qu'au fil du transfert - cette solidarité de la structure au transfert signe l'impossibilité de la réifier, c'est-à-dire de l'affranchir de l'Autre auquel elle se dévoile et, par là, de la réduire à ce qui ferait l'unité d'un tableau clinique, à la manière d'un endophénotype.

"L'actualité de la structure » peut donc s'entendre en au moins deux sens : (1) ce qui, au sein des savoirs, fait actualité et pour tout dire dissensus dans la structure entendue de manière freudienne, c'est qu'elle ne peut dessiner l'espace d'un déploiement subjectif qu'à faire intervenir son adresse - et que (2) c'est depuis cette adresse que doit être comprise son actualisation, sous peine de réduire celle-ci à la réalisation déterminée d'un programme prédéfini.

J'aimerais développer cette ligne argumentative à partir d'un contexte clinique où l'organique - et plus précisément le génétique, et ses effets neurodéveloppementaux - a une incidence incontestable sur le psychique, et où cette incidence est identifiée par la pédopsychiatrie biologique contemporaine à un facteur majeur de risque de psychose. Mon objectif ne sera pas de débattre directement avec ce paradigme biologique (voir à ce sujet les travaux de F. Gonon et, tout récemment, de loannidis, etc.) mais d'illustrer le sens et les effets du maintien d'une entente

\footnotetext{
Olivier Putois, psychologue clinicien, psychanalyste; Maître de conférences en Psychopathologie,

psychologie clinique et psychanalyse à l'Université de Strasbourg ; olivier.putois@gmail.

com

Texte réécrit d'une intervention faite aux Journées d'Espace analytique, Lacan, l'expé-

rience analytique, le 10 mars 2018.
} 
freudienne du réel de la structure, dans un contexte pourtant propice à l'adoption spontanée d'une position réaliste naïve où les accidents génétiques suffiraient à expliquer la mise en place d'une organisation psychotique - et interdiraient son évolution au fil des échanges avec l'Autre.

\section{Un tableau clinique sous-tendu par le risque de psychose?}

Je proposerai donc des remarques épistémologiques et cliniques sur les enjeux de la cure de patients infanto-juvéniles atteints d'un syndrome génétique à incidence neurodéveloppementale, dit syndrome de DiGeorge, ou syndrome de microdélétion 22q11.2, considéré par la neuropsychiatrie comme paradigmatique du risque génétique de psychose.

Quelques éléments médicaux d'abord sur ce syndrome ${ }^{1}$, afin de saisir en quoi il en est venu à constituer ces dernières années, pour la (pédo)psychiatrie biologique, un investissement massif $-\mathrm{y}$ compris au sens financier du terme. II s'agit d'un désordre génomique (la délétion ou effacement d'une partie du chromosome 22) qui apparaît de novo dans la presque totalité des cas. C'est-à-dire que parmi les patients, souvent jeunes, chez qui la microdélétion a été mise en évidence par analyse génomique, la grande majorité d'entre eux n'ont pas hérité cette altération de leurs parents, qui n'en sont pas porteurs : elle est au contraire survenue dans leurs cellules sexuelles. Cela engage un certain rapport à l'enfant, placé sous le signe de l'étrangeté.

Les conséquences de ce désordre génomique au plan organique sont multiples-le tableau clinique comporte quasiment deux cents traits d'expression variable. Les traits isolés initialement sont liés au développement du cœur, de la face, du système immunitaire, et du système endocrine. Mais les traits les plus communs et les plus prégnants sont d'ordre neurodéveloppemental, et ce sont eux qui influencent le plus directement le psychisme-le plus directement car il va de soi que l'atteinte de la fente du palais, fréquente durant la croissance du fœtus, a une incidence majeure sur la sphère de l'oralité.

L'atteinte neurologique se manifeste notamment par une altération dans le développement du cortex ; par exemple, une étude récente de volumétrie cérébrale

1. On en trouvera davantage dans R. Potier, O. Putois et coll., « Regards croisés sur une pathologie développementale d'origine génétique. Réflexions épistémologiques et cliniques sur l'impact psychique du syndrome de microdélétion 22q11.2 », Topique, $\mathrm{n}^{\circ} 134$, p. 103-117. 
chez l'enfant met en évidence une corrélation significative entre le DiGeorge et une altération dans le développement du cortex dorsolatéral préfrontal. Pour le dire dans les termes du paradigme cognitif qui règle la recherche en neurologie sur le DiGeorge, c'est vraisemblablement l'atteinte de cette zone du cortex qui explique l'altération, constatée par des tests, de capacités comme l'attention, la mémoire de travail, la planification, le raisonnement et l'exécution - bref, un retard mental modéré. Évidemment, l'utilisation de tests d'évaluation standardisés pose des problèmes méthodologiques énormes, ne serait-ce qu'en raison de l'effet massif du dispositif sur le narcissisme fragile de ces jeunes patients ; je ne fais ici que donner quelques éléments du discours de la clinique psychiatrique, pour poser le contexte.

Mais la raison pour laquelle ce syndrome génétique mobilise l'attention et les financements de la neuropsychiatrie infanto-juvénile tient à ce qu'il constitue le paradigme de la recherche sur les biomarqueurs (indices organiques) du risque de psychose, également appelé "vulnérabilité psychotique ${ }^{2}$ ".

On le sait, de nombreuses études cliniques et épidémiologiques visent, depuis une vingtaine d'années, l'identification et la détection précoce de facteurs de risques associés à l'émergence de symptômes psychotiques, en particulier schizophréniformes. Dès lors, la mise en évidence statistique, chez les patients porteurs d'un DiGeorge, d'une prévalence plus haute de ce que le DSM appelle troubles de la personnalité schizotypique (anxiété sociale, besoin d'isolement, etc.) ou schizoïde, constituerait une victoire pour les modèles génétiques et neurodéveloppementaux de la schizophrénie. En effet, en cas de corrélation gène/trouble de personnalité schizotypique, on tiendrait un argument pour l'existence d'un endophénotype - c'est-à-dire une réalité organique, intermédiaire entre le génome et le psychique, où s'ancrerait le risque d'émergence de la schizophrénie.

On comprend donc pourquoi ce syndrome mobilise tant d'énergie et de fonds du côté de la recherche en pédopsychiatrie biologique : celle-ci voit dans le DiGeorge la possibilité de réaliser le programme constitutionnaliste de la psychiatrie $d u X X^{e}$ siècle, incarné par exemple par la diathèse héréditaire chère à Charcot.

Or, au plan sémiologique, le style relationnel des petits sujets atteints d'un DiGeorge présente fréquemment, dès 7-8 ans, un retrait social, des manifestations fortes d'anxiété, une certaine timidité, des difficultés à engager l'échange

2. Je reprends dans ce passage des éléments d'un article commun : S. Troubé, R. Potier, O. Putois, "Remarques épistémologiques et cliniques sur la notion de vulnérabilité psychotique ", Neuropsychiatrie de l'enfance et de l'adolescence, vol. 66, n 4, p. 256-262. 

avec l'autre. (Ces traits ont pu, durant les premiers moments de la recherche sur ce syndrome, mener à le considérer comme une forme modérée de trouble du spectre autistique).

La question centrale des neuropsychiatres à partir de ce constat sémiologique a donc été de savoir si ce retrait et cette anxiété ne constituaient pas l'indice d'un risque statistique de schizophrénie infanto-juvénile, qu'expliquerait la microdélétion génétique. C'est pour cette raison que des recherches épidémiologiques ont été menées récemment sur la prévalence de psychoses au sein de cette population.

D'abord, sur un échantillon de plusieurs centaines de patients, des équipes notamment de Genève ont mis en évidence, chez un tiers des sujets, lors de la préadolescence, la survenue d'un trouble de la personnalité de type schizophréniforme-dans la nosographie du DSM. L'étape suivante a été d'interroger, dans une logique prédictive, les signes précurseurs de ce devenir-schizophrène. Or, on trouve une plus forte prévalence de troubles anxieux chez les patients porteurs d'un DiGeorge ayant développé une psychose infanto-juvénile. La conclusion est donc que la forte prévalence des troubles psychotiques chez les adolescents et préadolescents porteurs d'un DiGeorge pourrait se trouver liée à la fréquence des troubles anxieux dans leur enfance. Dès lors, la mise en évidence clinique de manifestations anxieuses chez un jeune sujet atteint d'un syndrome de DiGeorge fait immédiatement planer la menace du risque de psychose, qui sonne comme une prédiction destinale ; et ce, en dépit de la fragilité épistémologique de cette conclusion - car les troubles anxieux infantiles sont présents dans une grande variété de trajectoires développementales, et n'ont donc de ce fait pas de valeur prédictive, comme nous $\mathrm{y}$ insistons avec mes collègues mentionnés plus haut en note 2 .

\section{Deux réponses : risque de psychose vs désir de l'analyste}

Or, cette logique prédictive, incarnée dans la tonalité générale du discours médical qui informe les parents du risque de schizophrénie infantile, produit des effets iatrogènes massifs dans le fonctionnement familial, tant la crainte du déclenchement psychotique infiltre alors les rapports parentaux à l'enfant (comme angoisse de ce déclenchement). Ce qui accroît en retour le retrait relationnel de l'enfant, lequel se sent souvent d'autant plus identifié à ce signifiant - et ce, d'autant plus que même lors de l'adolescence, sa dépendance réelle le conduit à passer plus de temps avec ses parents. (Cette infiltration potentielle des rapports parentaux par le signifiant du risque de psychose doit être prise d'autant plus au sérieux 
qu'il est possible de diagnostiquer la présence de ce syndrome chez le fœus dès 10 semaines d'aménorrhée.)

En effet, ce risque de psychose devient fréquemment le signifiant qui mène à une demande de prise en charge, fut-elle analytique. Dans un tel contexte, comme le rappelle Mannoni ${ }^{3}$, il est nécessaire de se consacrer directement à l'incidence familiale du diagnostic de microdélétion, sans quoi la conduite de la cure sera rendue techniquement impossible du fait du masquage de la problématique parentale par le signifiant du diagnostic, qui empêche de déterminer la fonction inconsciente de la maladie de l'enfant (qui n'est pas son symptôme) pour les parents, et bloque l'évolution de l'enfant. Comme le disait G. Raimbault, suite à la conférence de Lacan sur "La place de la psychanalyse dans la médecine ", prendre en charge l'enfant exige d'abord de ressaisir le drame sous-jacent - c'està-dire majoritairement la fantasmatique parentale - qui donne sens au diagnostic au sein de la problématique familiale ${ }^{4}$.

L'efficace de la prise en charge dépendra en effet de la possibilité de suspendre ce signifiant diagnostique pour en faire un dehors, aussi bien pour les parents que pour l'enfant. Cette suspension est conditionnée par la capacité de l'analyste à s'extraire d'un effet de typification imaginaire (fantôme de l'endophénotype neuropsychiatrique), du fait du signifiant génétique qui risque d'obérer son écoute et de produire un sentiment d'impuissance plus ou moins masquée. C'est pourquoi Maud Mannoni rappelait que l'essentiel, dans les débuts des prises en charge de ce que la pédopsychiatrie appelle "pathologies avec retard mental et risque de psychose ", "c'est la nécessité pour le consultant de ne pas porter des diagnostics sans appel. L'enfant a toujours à gagner à ce qu'un maximum d'ouverture lui soit laissé ${ }^{5}$ ».

Le terme de «sans appel » est évidemment crucial car il identifie la reprise potentielle du signifiant diagnostic médical par l'analyste à un risque de gélification de l'enfant, désubjectivé car démétaphorisé, c'est-à-dire privé de l'adresse subjective qui permet de se structurer autour d'un symptôme afin de donner une signification subjective à ce signifiant - et aux éprouvés corporels qui l'accompagnent.

3. M. Mannoni, L'enfant arriéré et sa mère, Paris, Le Seuil, 1964, p. 116.

4. Cette intervention se trouve dans J. Lacan, « La place de la psychanalyse dans la médecine » (1966), dans J. Aubry, Psychanalyse des enfants séparés, Paris, Flammarion, 2010, p. 366. Nous développons ce point dans R. Potier, O. Putois, «A Lacanian Approach to Medical Demand, with a Focus on Pediatric Genetics : a Plea for Subjectivization », Frontiers in Psychoanalysis, nov. 2018.

5. M. Mannoni, L'enfant arriéré et sa mère, op. cit., p. 135. 
Ainsi, dans le suivi d'une jeune préadolescente de 12 ans porteuse de la microdélétion et présentant des défenses phobiques massives et des traits psychotiques, une étape préliminaire a consisté à faire travailler les parents reçus en parallèle (divorcés, avec une importante différence d'âge) sur l'écart qui semblait séparer leurs ententes respectives de la maladie, afin de remétaphoriser ce signifiant et de mettre en évidence la possibilité de l'inscrire dans des chaînes signifiantes distinctes, voire incompatibles. La maladie de l'enfant ne devait pas jouer (c'était le risque) une fonction d'apaisement des différends parentaux, garante d'un front uni qui interdise toute conflictualisation. Maintenir cet écart entre les perspectives des parents a permis de dégager la possibilité d'une adresse, c'est-à-dire un espace de subjectivation, pour leur fille : chacun pouvait comprendre différemment sa maladie - y compris elle.

II ne s'agit pas de nier la réalité des déterminants organiques - au risque de rappeler des fondamentaux, Freud et Lacan insistent sur le non-sens d'une telle position ${ }^{6}$-, mais de pointer les effets iatrogènes de la gélification du psychique noué à l'organique dans les seuls signifiants d'un discours médical autonomisé. Car répondre à la demande de diagnostic médical et de prise en charge en termes de risque de psychose, c'est-à-dire en choisissant une réponse en forme de prédiction autoréalisante ${ }^{7}$, c'est dénier les effets potentiellement transformationnels de la réponse guidée par le désir de l'analyste, tout en orientant la structure vers la psychose.

Une première phase de thérapie de la jeune fille a gravité autour de la possibilité de trouver un objet transitionnel (qui s'est fixée sur le dessin, en lien avec un signifiant familial maternel), et caractérisée par un retrait inhibé et beaucoup de passivité, requérant une technique assez active. Une fois la dynamique du transfert appuyée sur cette médiation, un travail sur le narcissisme primaire parental a permis d'envisager la libération de leur fille des canons scolaires standard pour qu'elle puisse se réaliser à sa manière - poursuite de la désaliénation du signifiant «sans appel » de risque de psychose. Ce qui a débouché, quelques mois plus tard, sur leur proposition spontanée de lui proposer d'arrêter le méthylphénidate - prescription standard pour " éviter la psychose »-après consultation du pédopsychiatre. L'effet de la décision a été spectaculaire et a permis un important travail transitionnel sur le spéculaire, par figuration dessinée de fantasmes d'ingestion réciproque où elle

6. Respectivement dans la préface des Trois essais, et dans Le Séminaire III : Les psychoses. 7. Les travaux de François Ansermet ont contribué à sensibiliser la communauté analytique à ces questions. 
prenait clairement, activement, la direction des échanges. Ce qui rendait envisageable la question de la coupure, à l'horizon de la cure.

\section{Une réponse ouverte}

La forclusion de la contribution de la réponse à la structuration du réel inconscient sépare le réalisme naïf du réel tel que peut l'entendre la psychanalyse - lequel intègre la réponse qui lui est faite. C'est la raison pour laquelle, lorsque la structure n'est pas encore totalement établie et qu'il se présente un risque d'organisation psychotique lié à la déphallicisation de l'enfant en contexte de handicap génétique ${ }^{8}$, Mannoni indiquait que la forme que doit prendre le désir de l'analyste est l'ouverture. Lacan ne s'y est pas trompé : dans les Quatre concepts, lorsqu'il présente l'ouvrage de Mannoni sur L'enfant arriéré et sa mère, il rappelle l'importance d'interroger la « dimension psychotique ${ }^{9}$ " du rapport parental, et singulièrement maternel, à l'enfant - d'où l'inconscient de ce dernier tire son noyau. C'est-à-dire qu'il faut saisir le point où dans l'inconscient de l'enfant peut commencer cette dimension psychotique, le point où il peut se trouver déphallicisé et, ainsi, désinscrit de l'affiliation à un Nom-du-père. Laissant ouverte cette dimension psychotique, on a la chance de l'aider à se construire autour d'un symptôme, et par là d'envisager la question de la coupure. Chez la jeune patiente que nous évoquions, des traits œdipiens sont petit à petit apparus, sur le fond de la transitionnalisation ouverte par l'apaisement des conflits parentaux qui s'étaient cristallisés sur le signifiant de microdélétion avec risque de psychose.

Ces considérations sur l'ouverture ne me paraissent pas locales, mais retrouvent à mon sens l'avertissement freudien sur le diagnostic au sens analytique, appréhension sous transfert du réel de la structure : la temporalité incompressible de son déploiement conduisait Freud à indiquer, en somme, que l'ouverture est une règle de conduite nécessaire au potentiel transformationnel de la cure. II écrivait en effet dans les Nouvelles conférences:

« Nos diagnostics n'interviennent très fréquemment qu'après coup, ils sont de la même espèce que l'épreuve des sorcières du roi d'Écosse, que j'ai lue dans Victor Hugo. Ce roi affirmait être en possession d'une méthode infaillible pour reconnaître une sorcière. II

8. Nous en traitons dans notre article L. Razon, O. Putois, A. Vanier, « The Lacanian Concept of Cut in Light of Lacan's Interactions with Maud Mannoni », Frontiers in Psychoanalysis, 2017.

9. J. Lacan, Le Séminaire, Livre XI, Les quatre concepts fondamentaux de la psychanalyse (1964), Paris, Le Seuil, 1971, p. 215. 
la faisait cuire dans une bassine d'eau bouillante, et puis il goûtait la soupe. Après quoi, il pouvait dire : "c'était une sorcière" où "non, ce n'en était pas une". II en va de même pour nous, sauf que c'est nous qui sommes lésés. Nous ne pouvons pas juger le patient qui vient pour un traitement, pas plus que le candidat qui vient pour une formation, avant de l'avoir étudié analytiquement pendant quelques semaines ou mois ${ }^{10}$. »

\section{RÉSUMÉ}

La spécificité de l'entente freudienne de la structure tient à son inclusion indissoluble de l'Autre, qui la constitue en son adresse subjectivante en contribuant à lui donner forme de demande. Dans un contexte médical avéré comme celui du syndrome de DiGeorge (microdélétion 22q11.2), maladie génétique comprenant un fort versant neurodéveloppemental, l'enjeu matriciel d'une praxis analytique tient d'abord à la préservation de cette adresse, à l'encontre d'une réduction de la structure à un endophénotype, réalité organique autonome introduite pour expliquer ce que l'épidémiologie psychiatrique présente comme risque statistique de psychose associé au syndrome. En pointant les risques d'effets iatrogènes autoréalisants de ce signifiant de risque psychotique jusque dans la demande parentale de prise en charge analytique, on propose des pistes pour soutenir la subjectivation de l'enfant par le désir de l'analyste en désaliénant en parallèle la famille de ce signifiant, jusque dans le travail sur les traitements.

\section{MOTS-CLÉS}

Demande, syndrome de DiGeorge, diagnostic, risque de psychose, psychothérapie analytique, famille.

\section{SUMMARY}

Responding to the (question of) psychic structure in the context of genetic diseases

Structure, understood in a Freudian fashion, includes the dimension of the Other. This dimension shapes it into a demand, which as an address is the matrix of subjectivization. In a medical context such as that of the DiGeorge syndrome (microdeletion 22q11.2 syndrome), a genetic disease with a strong neurodevelopmental impact, the central stake of psychoanaIytic practice is primarily to preserve this dimension of address, in order to prevent the reduction of structure to an endophenotype, i.e. an autonomous organic reality introduced to account for what epidemiological psychiatry views as a statistical risk of psychosis associated with 22q11.2 syndrome. By pointing the risks of self-realizing iatrogenic effects induced by this signifier of psychotic risk - which motivates many parental demands of analytic work - we propose a few threads to support the child's subjectivization through the desire of the analyst while, in parallel, desalienating the family from this signifier (which also pervades medication protocols).

\section{KEYWORDS}

Demand, DiGeorge syndrome, diagnosis, psychotic risk, psychoanalytic psychotherapy, family.

10. S. Freud, « XVII" leçon : Éclaircissements, applications, orientations », dans OCF.P, t. XIX, Paris, Puf, 2004, p. 240. 